\title{
Molecularly imprinted silica gel incorporated with agarose polymer matrix as mixed matrix membrane for separation and preconcentration of sulfonamide antibiotics in water samples
}

\begin{abstract}
Molecularly imprinted silica gel (MISG) was incorporated through dispersion in agarose polymer matrix to form a mixed matrix membrane (MMM) and was applied for the determination of three sulfonamide antibiotic compounds (i.e. sulfamethoxazole (SMX), sulfamonomethoxine (SMM), and sulfadiazine (SDZ)) from environmental water samples. Several important microextraction conditions, such as type of desorption solvent, extraction time, amount of sorbent, sample volume, $\mathrm{pH}$, and effect of desorption time, were comprehensively optimized. A preconcentration factors of $\geq 20$ was achieved by the extraction of $12.5 \mathrm{~mL}$ of water samples using the developed method. This microextraction-HPLC method demonstrated good linearity $(1-500 \mu \mathrm{g} L-1)$ with a coefficient of determination (R2) of 0.9959-0.9999, low limits of detection $(0.06-0.17 \mu \mathrm{g} \mathrm{L}-1)$ and limits of quantification $(0.20$ $0.56 \mu \mathrm{g} L-1)$, good analyte recoveries (80-96\%), and acceptable relative standard deviations $(<10 \%)$ under the optimized conditions. The method is systematically compared to those reported in the literature.
\end{abstract}

Keyword: Sulfonamide antibiotics; Molecularly imprinted silica gel; Mixed matrix membrane; High performance liquid chromatography; Environmental water 